\title{
Inactivation du gène VHL (von Hippel-Lindau) et tumorigenèse : un rôle essentiel du TGF $\alpha$
}

La maladie de von Hippel-Lindau (VHL) se caractérise au plan clinique par une prédisposition au développement de multiples tumeurs particulièrement vascularisées notamment au niveau des reins, du cerveau et de la rétine. Cette phacomatose héréditaire transmise en dominance est due à des mutations inactivatrices $\mathrm{du}$ gène suppresseur de tumeur VHL. L'apparition des tumeurs est la conséquence d'un événement mutationnel somatique portant sur l'allèle $\mathrm{VH} L$ sauvage et conduisant à une inactivation bi-allélique de $\mathrm{VH} L$, ce mécanisme étant en accord avec I'hypothèse, formulée par Knudson au sujet des gènes suppresseurs de tumeurs, de deux événements mutagéniques successifs (two-hits). Des mutations dans le gène $\mathrm{VH} L$ sont aussi présentes dans la plupart des carcinomes sporadiques à cellules claires du rein ( $R C C)$, la pathologie maligne du rein la plus fréquente [ 1 , 2]. La réintroduction de VHL dans ces cellules tumorales, nommées $\mathrm{VH}^{-1-} \mathrm{RCC}$, supprime la croissance de la tumeur in vivo [3], suggérant que VHL joue un rôle de "gardien » (gate-keeper) dans le contrôle de la prolifération cellulaire (ou dans celui de la mort cellulaire) [4].

La protéine VHL est composée de 213 acides aminés. Elle forme avec quatre autres protéines, les élongines $B$ et $C$, la culline 2 et rbx- 1 , un complexe E3 ubiquitine protéine ligase permettant l'ubiquitinylation de protéines qui seront ensuite dégradées par le protéasome $26 \mathrm{~S}$ (m/ s 1999, $n^{\circ} 8-9$, p. 1051). Un des substrats de VHL est la sous-unité alpha du facteur de transcription HIF (hypoxiainducible factor) [5]. Si, dans des dégradé de façon constitutive par le complexe VH L, il est en revanche stabilisé en conditions hypoxiques, et $s^{\prime}$ associe alors avec HIF $\beta$ pour former le complexe de transcription HIF. Ce dernier provoque la transcription des gènes induits par I'hypoxie comme celui codant pour le facteur angiogénique VEGF (vascular en dothelial growth factor). Dans les cellules $\mathrm{VH} \mathrm{L}^{-1}$ RCC, la dégradation de HIF $\alpha$ ne se produit pas, ce qui entraîne une surexpression constitutive des gènes normalement contrôlés par HIF indépendante des tensions d'oxygène. La surexpression de facteurs angiogéniques peut facilement expliquer le caractère particulièrement vascularisé des tumeurs dont le gène VHL est inactivé, mais il paraît plus difficile d'envisager qu'elle soit à l'origine d'un processus tumoral initial dont les mécanismes sont encore inconnus.

C'est pourquoi plusieurs équipes ont tenté d'identifier, dans les cellules VH L ${ }^{-I-R C C}$, des défauts de croissance susceptibles d'être corrigés par la réintroduction de VHL. Nous avions précédemment montré que des cellules $\mathrm{VH} \mathrm{L}^{-1-R C C}$, cultivées à faible confluence, ne s'arrêtaient pas de croître en l'absence de sérum [6], ce qui est une des caractéristiques typiques des cellules cancéreuses. $L a$ réintroduction de VH L permettait de rendre à nouveau la croissance cellulaire dépendante de la présence de sérum, suggérant un rôle pour VHL dans le contrôle du cycle cellulaire. Or, les cellules $\mathrm{VH}^{-1-R C C}$ peuvent quand même croître dans des conditions d'inhibition par contact [6]. Ceci permettait d'évoquer qu'un facteur sécrété en excès par ces cellules soit responsable des anomalies de croissance observées à faible densité cellulaire. Une des molécules produites en excès par les cellules RCC en culture et dans les tumeurs RCC in vivo est le facteur de croissance TGF $\alpha$ (transforming growth factor alpha). Des études récentes ont en effet révélé que l'expression du gène codant pour le TGF $\alpha$, comme celle du gène codant pour le VEGF, est contrôlée par VHL [7] et l'oxygène [8]. Contrairement aux autres facteurs produits en excès par les celIules VH L ${ }^{-1}$-RCC, comme le VEGF ou le TGF $\beta$, le TGF $\alpha$ est capable de stimuler la prolifération de cellules épithéliales de tubule proximal de rein [8], les cellules qui sont très probablement à l'origine des RCC. On sait que l'action du TGF $\alpha$ sur la croissance cellulaire est due à l'activation du récepteur de I'EGF (EGF-R pour epidermal growth factor receptor), et nous avons donc émis I'hypothèse selon laquelle un signal de croissance autocrine impliquant le TGF $\alpha$ serait l'événement causal des anomalies de croissance des cellules VH L ${ }^{-1-R C C}$. Effectivement, l'inhibition de la voie TGF $\alpha /$ EGF-R est suffisante pour abolir les capacités des cellules VH L-I-RCC à proliférer en l'absence de sérum [8], et à former des tumeurs lorsqu'elles sont injectées à des souris immunodéficientes [10]. $L$ 'inactivation de cette voie autocrine TGF- $\alpha$ /EGF-R ne corrige pas, en revanche, les autres défauts caractéristiques de ces cellules cancéreuses que sont les dépôts de fibronectine [9] et la stabilisation constitutive de $H I F \alpha$. Ainsi, même si le rôle d'autres stimulus de prolifération ne peut être formellement exclu, il apparaît que le blocage de la voie du TGF $\alpha$ permet, à elle seule, d'inhiber la prolifé- 
ration de ces cellules cancéreuses, tout comme la réintroduction $\mathrm{du}$ gène $V H L$ sauvage.

Ces résultats nous permettent de proposer un modèle de développement des RCC selon lequel la perte de fonction de $V H L$, en stabilisant $H I F \alpha$, stimule la transcription de gènes, comme ceux codant pour le VEGF ou le TGF $\alpha$, qui ne sont normalement exprimés que dans des conditions d'hypoxie (figure 1). Même s'il n'a pas été montré formellement que le gène codant pour le TGF $\alpha$ est directement induit par HIF, ceci est fort probable puisque son expression est augmentée par I'hypoxie et que son promoteur contient une séquence consensus de réponse à l'hypoxie, HRE (hypoxia-res- ponse element), connue pour lier le complexe HIF. Cette production excessive de TGF $\alpha$ serait responsable, via l'activation du récepteur EGF-R, de la croissance inappropriée des cellules épithéliales rénales, et pourrait être l'événement initial du processus tumoral.

Les RCC étant des cancers particulièrement résistants aux thérapeutiques anticancéreuses habituelles comme la radiothérapie et la chimiothérapie, le développement de nouvelles approches thérapeutiques semble représenter la seule possibilité de traiter les patients atteints. L'utilisation d'un des nombreux antagonistes de EGF-R, dont certains sont déjà en cours d'essai clinique, pourrait représenter un réel espoir pour ces patients.

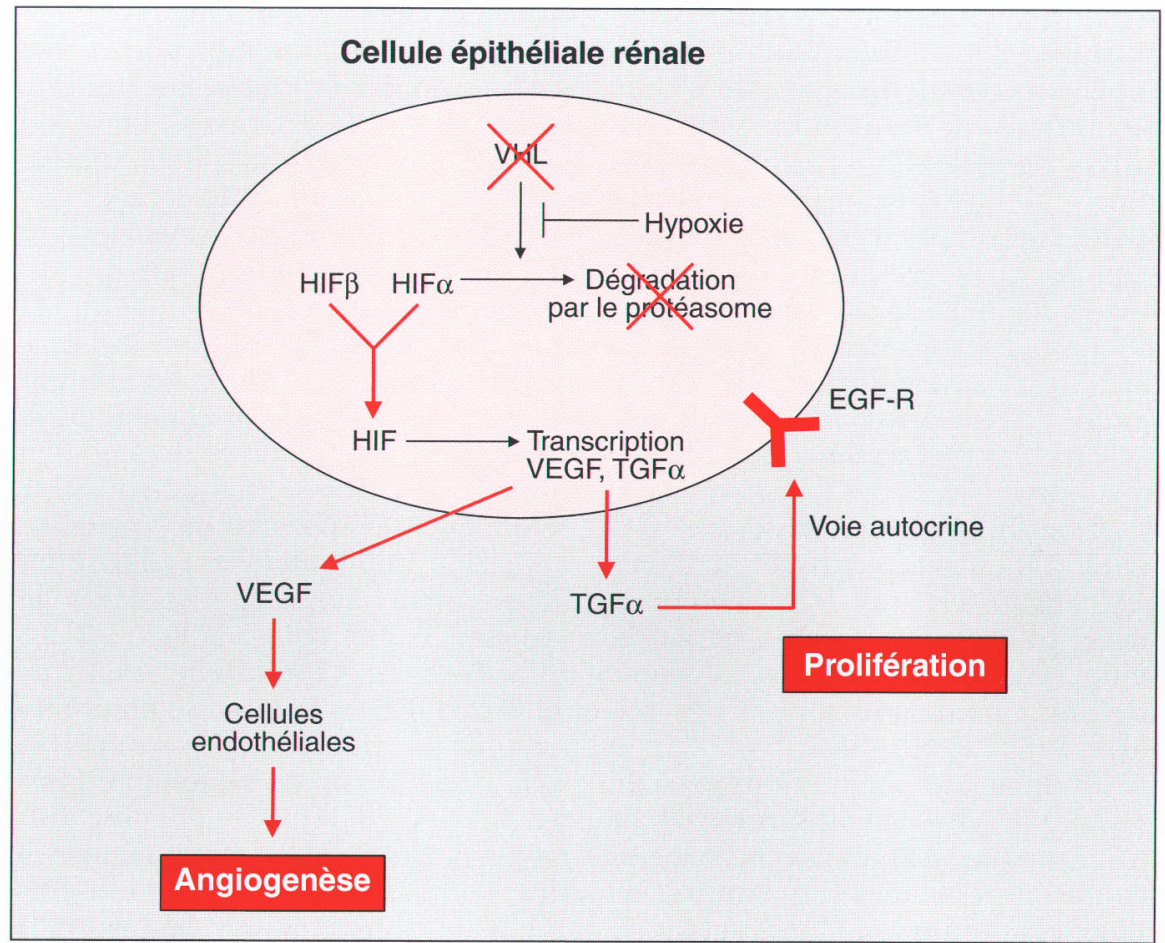

Figure 1. Mécanisme d’action de VHL dans les cellules épithéliales de tubule proximal de rein. La protéine VHL provoque, en conditions normales, la dégradation de HIF $\alpha$ par le protéasome. En condition d'hypoxie, cette dégradation est inhibée, et HIF $\alpha$ peut se lier à HIF $\beta$ pour former le facteur de transcription actif, HIF, qui stimule l'expression des gènes codant pour le TGF $\alpha$ et le VEGF. En I'absence de VHL (tumeurs $\mathrm{VHL}^{-/-} \mathrm{RCC}$ ), HIF $\alpha$ est stabilisé de façon indépendante de toute hypoxie, et il existe une surexpression constitutive du TGF $\alpha$ et du VEGF. Le TGF agit alors de façon autocrine, en activant le récepteur de l'EGF, provoquant ainsi la prolifération des cellules épithéliales rénales, tandis que le VEGF stimule l'angiogenèse.
1. Latif F, Tory K, Gnarra J, et al. Identification of the von Hippel-Lindau disease tumor suppressor gene. Science 1993; 260: 1317-20.

2. Gnarra JR, Tory $K$, Weng $Y$, et al. Mutations of the VHL tumor suppressor gene in renal carcinoma. Nat Genet 1994; 7: 85-90.

3. Iliopoulos 0 , Kibel A, Gray S, Kaelin WG. Tumor suppression by the human von-Hippel Lindau gene product. Nat M ed 1995; 1: 822-6.

4. Kinzler KW, Vogelstein B. Cancer susceptibility genes. Gatekeepers and caretakers. Nature 1995; 386: 761-3.

5. Maxwell P, Weisner M, Chang GW, et al. The von Hippel-Lindau gene product is necessary for oxygen dependent proteolysis of hypoxia inducible factor $\alpha$ subunits. Nature 1999; 399: 271-5. 6. Pause A, Lee S, Lonergan KM, Klausner RD. The von $\mathrm{H}$ ippel-Lindau tumor suppressor gene is required for cell cycle exit upon serum withdrawal. Proc Natl A cad Sci USA 1998; 95: 993-8.

7. Knebelmann B, Ananth $\mathrm{S}$, Cohen $\mathrm{H}$, Sukhatme V. Transforming growth factor $\alpha$ is a target for the von Hippel-Lindau tumor suppressor. Cancer Res 1998; 58: 226-31.

8. de Paulsen N, Brychzy A, Fournier MC, et al. Role of transforming growth factor-alpha in von Hippel-Lindau $(\mathrm{VHL})^{-1-}$ clear cell renal carcinoma cell proliferation: a possible mechanism coupling VHL tumor suppressor inactivation and tumorigenesis. Proc Natl Acad Sci USA 2001: 98: 1387-92.

9. Ohh M, Yauch RL, Lonergan KM, et al. The von $H$ ippel-Lindau tumor suppressor protein is required for proper assembly of an extracellular fibronectin matrix. Mol Cel 1998; 7: 959-68.

10. Prewett M, Rothman M, Waksal H, Feldman $\mathrm{M}$, Bander NH, Hicklin DJ. Mouse-human chimeric anti-epidermal growth factor receptor antibody C225 inhibits the growth of human renal cell carcinoma xenografts in nude mice. Clin Cancer Res 1998; $4: 2957-66$.

\section{Natalie de Paulsen Isabelle Groulx Stephen Lee*}

Département de médecine moléculaire et cellulaire et Centre de recherche sur les maladies du rén, Faculté de médecine, Université d'0ttawa, Ottawa, Ontario, Canada. K1H 8M 5.

email : sle@uottawa.ca 